\title{
TORRE PARA COMUNICACIONES Y PRÁCTICAS DE BOMBEROS EN LA ELIANA, VALENCIA. ESPAÑA
}

\author{
(COMMUNICATION AND FIRE BRIGADE PRACTICE TOWER IN ELIANA, VALENCIA. SPAIN)
}

José Monfort Lleonart, Arquitecto

E.T.S. de Arquitectura de Valencia

\section{RESUMEN}

Se describen las caracteristicas de proyecto y ejecución de una torre de comunicaciones, que además debe servir para realizar prácticas de bomberos y que alberga en su base un depósito de agua.

\section{SUMMARY}

The article brings the description of the characteristics of the project and the execution of the work on a communication tower which should also serve for practices of fire brigades and which has a water tank in the base.

\section{INTRODUCCIÓN}

Dentro del programa de Seguridad/Protección Civil de la Generalitat Valenciana, sobre una parcela situada en el término municipal de La Eliana, se han construido unos edificios para Escuela de Policias y Bomberos, Centro Informático y de Comunicaciones, y Parque de Bomberos, y se proyecta para un futuro inmediato la construcción de naves para almacenamiento y laboratorios, galeria de tiro, gimnasio, helipuerto y aparcamiento de vehículos.

El Centro Informático y de Comunicaciones necesitaba una torre para albergar tres antenas parabólicas de rejilla, que, dado el amplio programa de seguridad a desarrollar en esta parcela, se planteó que pudiera servir también para realizar prácticas de bomberos; en el programa se incluia también un depósito de agua, con una capacidad útil no menor de $150 \mathrm{~m}^{3}$, para las necesidades de todo el conjunto, cuya base debía quedar al nivel del terreno.

Las características del proyecto y construcción de es. ta torre, son a las que hacemos referencia en este artículo.

\section{CONDICIONES DE PROYECTO}

Como datos de partida para la torre, se pedia:

- Sustentar tres parábolas, dos de ellas de $2 \mathrm{~m}$ de diámetro y la otra de $3 \mathrm{~m}$, esta última situada a una altura de $45 \mathrm{~m}$ y las otras dos a alturas no definidas en el momento de proyecto, pero siempre por encima de los $20 \mathrm{~m}$.

- El cálculo en condiciones de resistencia debía efectuarse para la presión del viento correspondiente a una velocidad de $200 \mathrm{~km} / \mathrm{hora}$.

- En condiciones de servicio, para la presión del viento correspondiente a una velocidad de $100 \mathrm{~km} / \mathrm{ho}$ $\mathrm{ra}$, el giro del eje de orientación de las parábolas no debia ser superior a 0,125 grados.

- No se establece ninguna prescripción con respecto a las acciones sismicas.

- La zona de prácticas de bomberos debía alcanzar hasta una altura de $24 \mathrm{~m}$, con plataformas horizontales cada $3 \mathrm{~m}$, y cerramientos laterales que permitieran realizar descenso con escaleras colgadas y cuerdas. 


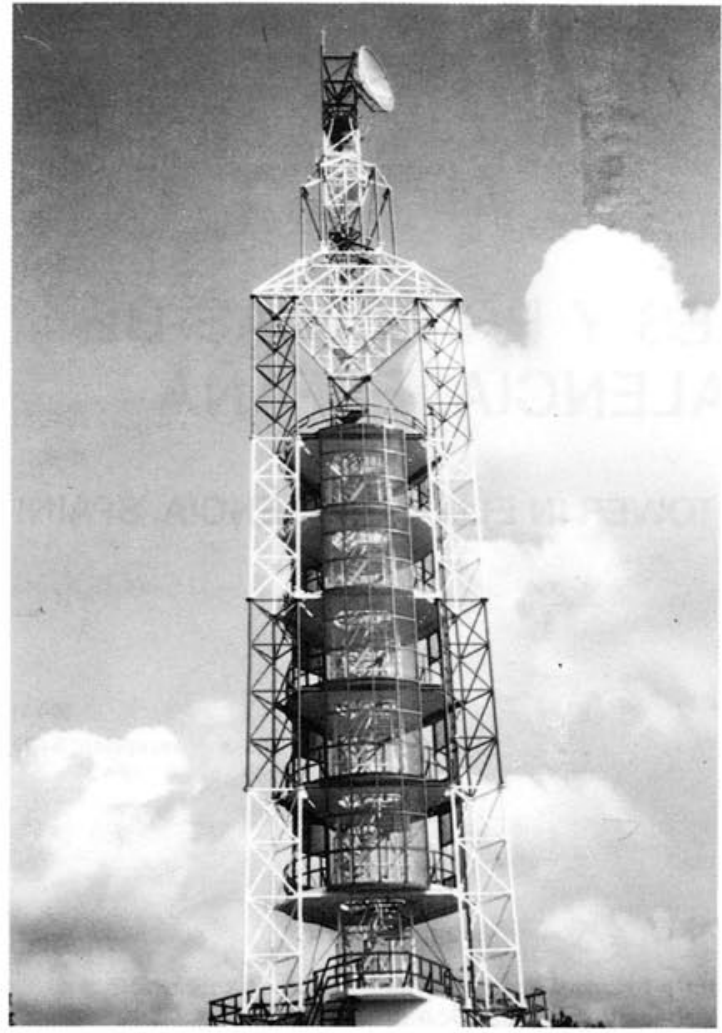

Fig. 1.-Vista general.

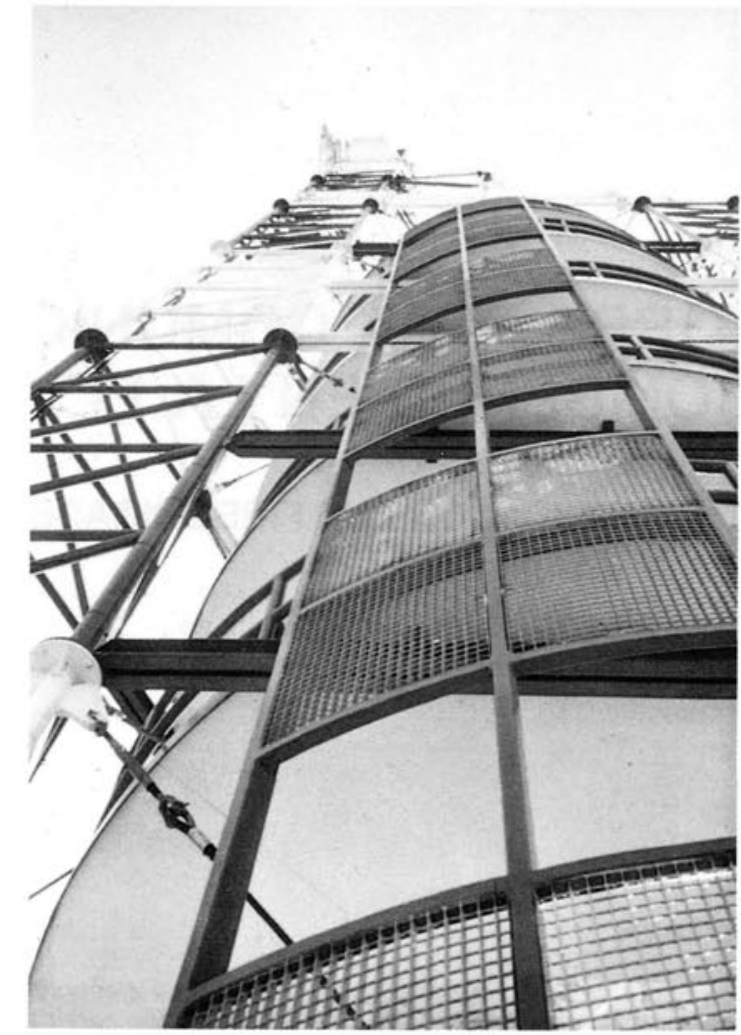

Fig. 2.-Detalle cerramientos laterales de las plataformas.

\section{ASPECTOS CONSTRUCTIVOS}

A partir de estos datos, buscando una solución estructural que tenga suficiente rigidez a flexión y torsión para cumplir las restricciones impuestas, se han proyectado tres soportes triangulares en celosia, de $1,50 \mathrm{~m}$ de lado cada uno, que a su vez van enlazados entre sí por tirantes que diagonalizan el conjunto, $y$ hacen que su comportamiento estructural sea el de una ménsula en celosia de sección triangular de 7,50 m de lado en su parte inferior, con reducción gradual de esta sección en la parte alta (figuras 1 y 3).

El hueco central que queda entre las tres celosias, que constituyen los soportes del conjunto, se aprovecha para colocar plataformas circulares de hormigón cada $3 \mathrm{~m}$ de altura que sirvan para realizar las prácticas de bomberos, hasta una altura de $24 \mathrm{~m}$ (figura $5 \mathrm{~d}$ ). Estas plataformas van apoyadas sobre tres vigas que unen los soportes principales, y hacen de montantes en el conjunto de la celosia (figura 7); lateralmente van cerradas por paneles de rejilla metálica galvanizada que simulan muros, con huecos, por los que realizar prácticas de descenso vertical (figura 2). El resto del perímetro de la plataforma va limitado por un antepecho metálico, simulando balcones, por los que realizar prácticas con escalera colgada.

c Consejo Superior de Investigaciones Cientííicas

Licencia Creative Commons 3.0 España (by-nc)
A partir de la altura de $30 \mathrm{~m}$, los tres soportes de celosía van enlazados por vigas de sección rectangular, también de celosia, que rigidizan este nivel, y desde aqui, en altura, sigue solamente uno de los soportes hasta alcanzar la cota donde se deben ubicar las parábolas. Para conseguir la rigidez necesaria en este último tramo, se ha proyectado una celosia de transición a partir de la cota $31,50 \mathrm{~m}$, que disminuye la rigidez de forma gradual. Todo el trazado geométrico se ha realizado siguiendo una retícula de triángulos equiláteros de $1,50 \mathrm{~m}$ de lado.

En la parte baja del conjunto, hasta los $6 \mathrm{~m}$ de altura, en el hueco entre los soportes de celosía se ha ubicado el depósito de agua, con capacidad aproximada de $175 \mathrm{~m}^{3}$ útiles, delimitado por muros y losas de hormigón (figuras 5 a y b). En la base de los soportes, adosado al muro del depósito, se realizaron tres macizos de hormigón armado de sección triangular (aligerado interiormente) hasta una altura de $4,50 \mathrm{~m}$, desde los que arrancan los elementos metálicos convenientemente anclados (figura 8). Rodeando este depósito, con una altura de $3 \mathrm{~m}$ sobre el nivel del suelo, se han proyectado unos recintos que, además de configurar la base de la torre, se destinan a sala de máquinas del depósito y para realizar prácticas con humo los bomberos (figura $5 \mathrm{a}$ ); el trazado en planta de todos estos elementos de base se ha realizado siguiendo la misma trama triangular antes citada. 

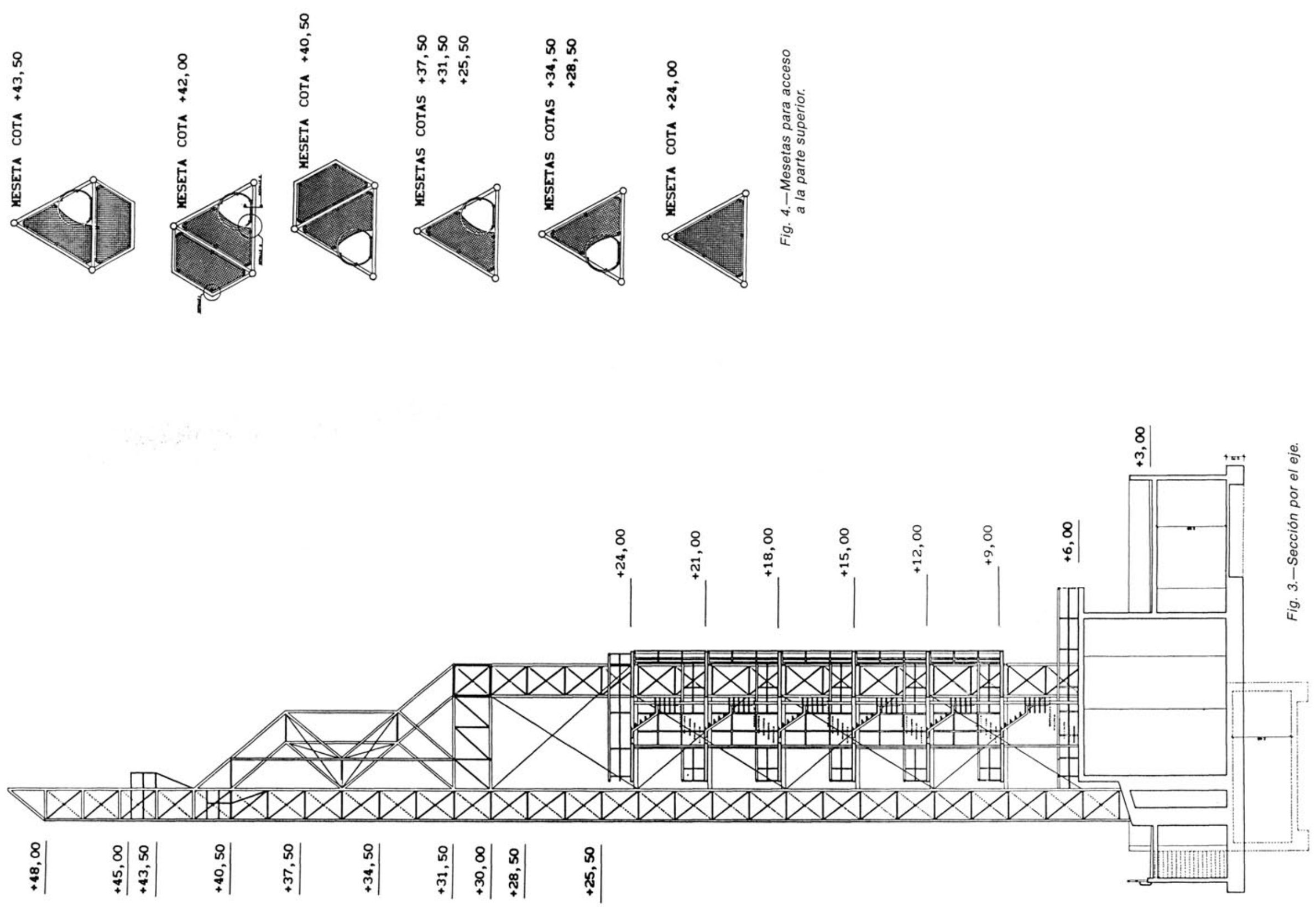


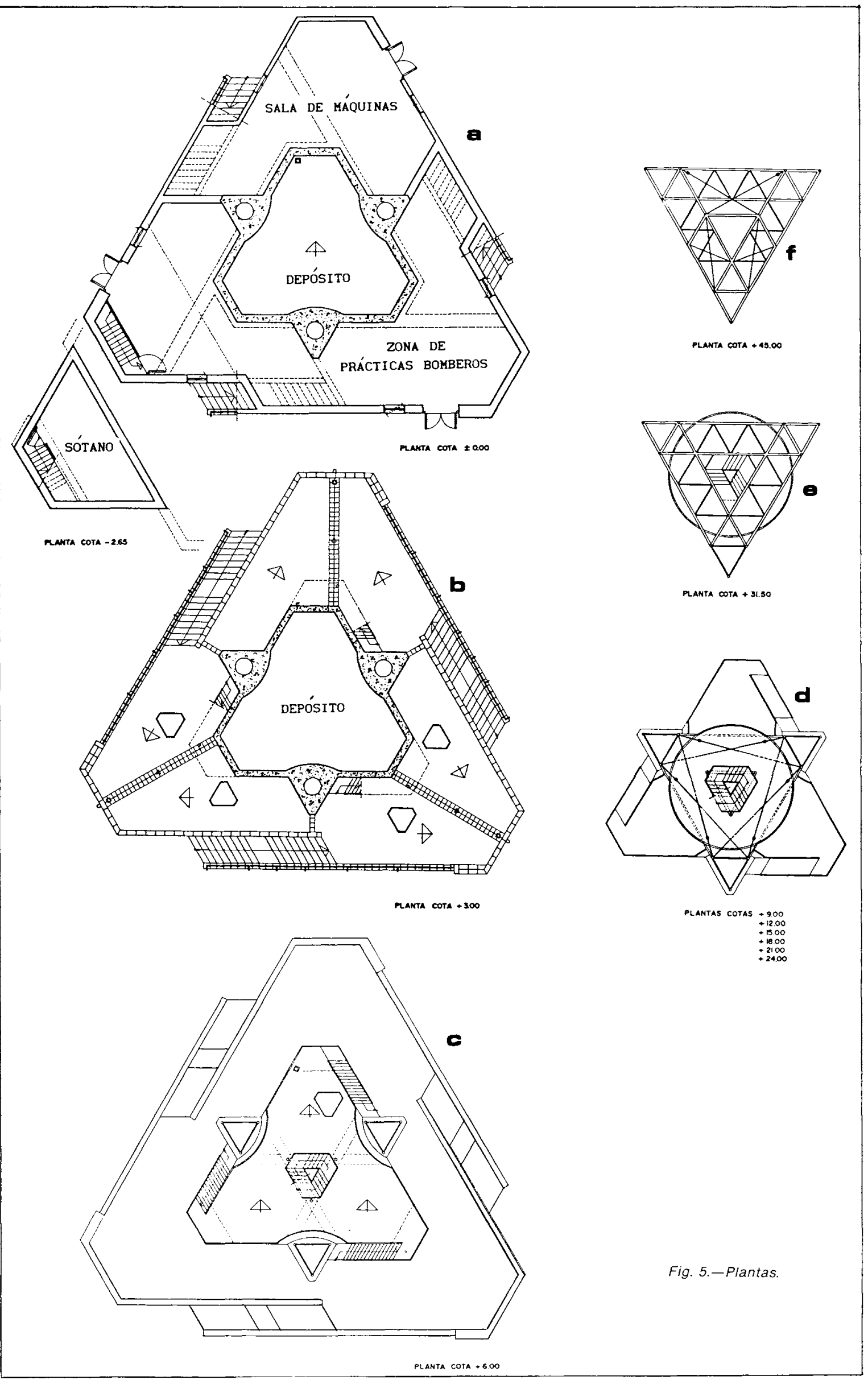




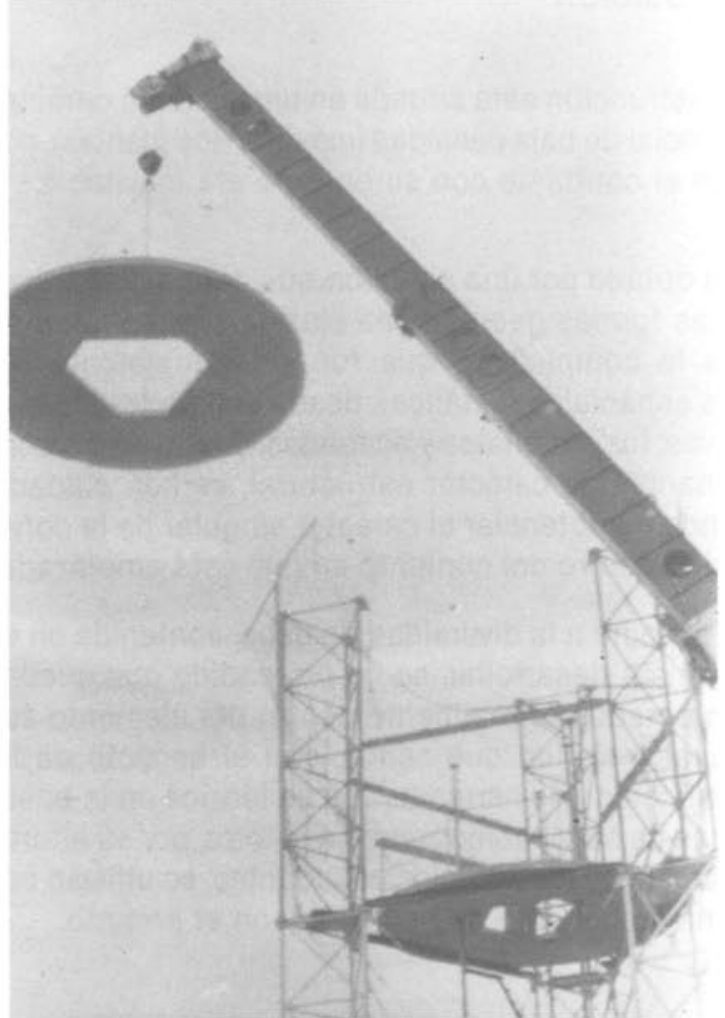

Fig. 6.-Elevación de las plataformas de hormigón construidas en el suelo.

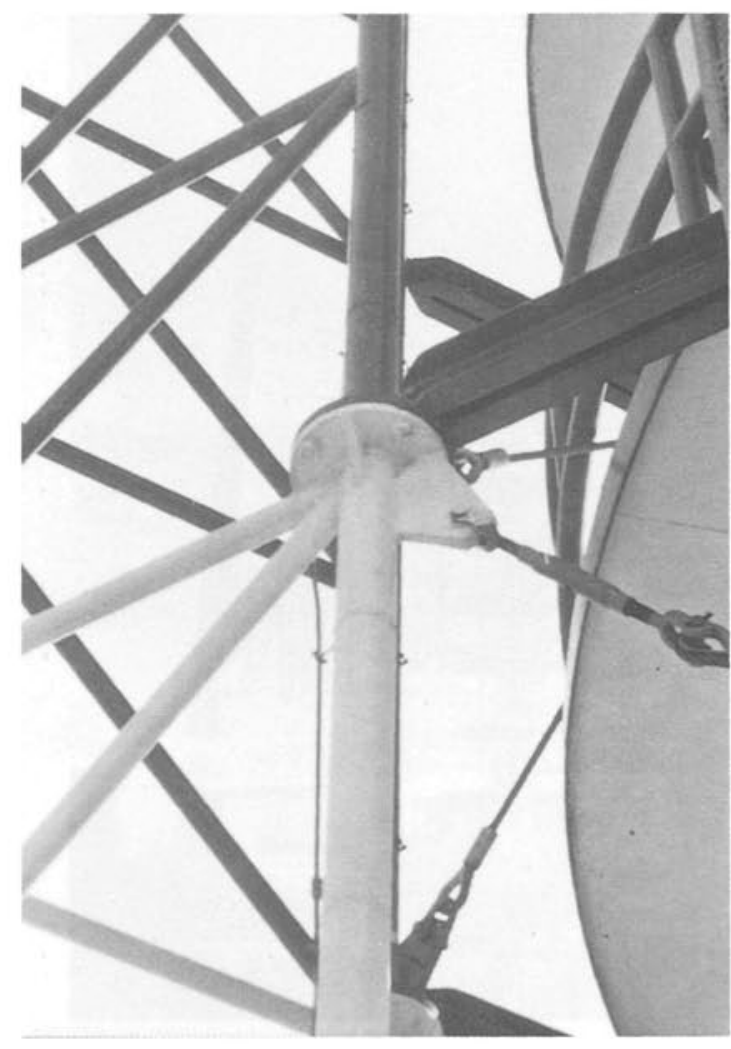

Fig. 7.-Detalle de nudo intermedio en zona de plataformas.

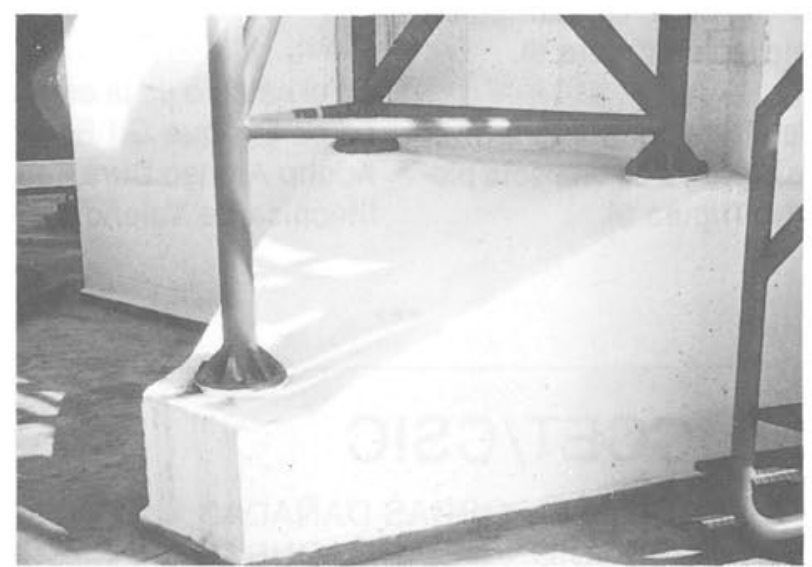

Fig. 8.-Anclaje de la estructura metálica en el macizo de hormigón a la cota $+4,50 \mathrm{~m}$

El acceso a todos los niveles se realiza por medio de escaleras; las plataformas de prácticas de bomberos disponen de una escalera central de acceso relativamente cómodo hasta la última de ellas, a $24 \mathrm{~m}$ de altura. A partir de aqui, para el acceso a las parábolas, se ha proyectado una escalera vertical por el interior del soporte triangular, con mesetas intermedias de seguridad que se amplian por el exterior del soporte en el nivel que se ha previsto la colocación de las parábolas, para facilitar la accesibilidad (figura 4).

\section{MONTAJE}

La estructura metálica se ha proyectado a base de tubo redondo galvanizado, con uniones soldadas y atornilladas (figura 7): se realizaron en taller tramos soldados de cada uno de los soportes triangulares de $6 \mathrm{~m}$ de longitud, que posteriormente se ensamblaron en obra por medio de tornillos de alta resistencia; todo el conjunto se pintó posteriormente con poliuretano en 


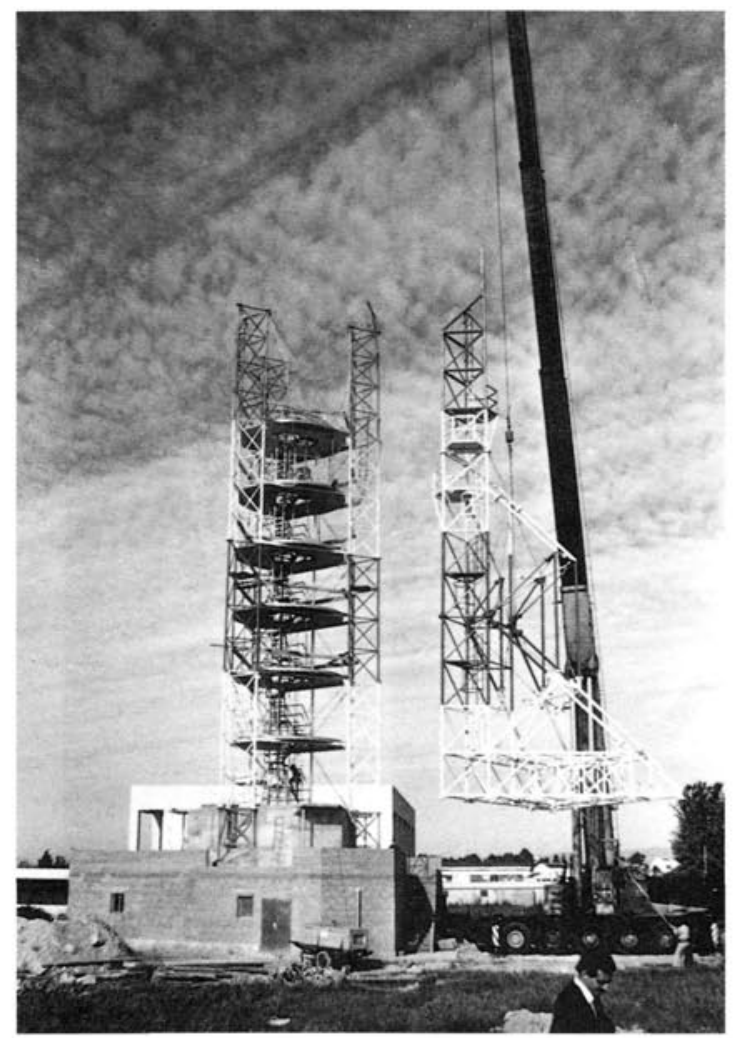

Fig. 9.-Montaje del tramo superior.

franjas blancas y rojas, según las Recomendaciones de la OACl. Las losas de hormigón se ejecutaron en el suelo, para su posterior elevación (figura 6).

El tramo superior de $18 \mathrm{~m}$ de altura se montó a nivel de suelo y, posteriormente, se elevó ya de una sola pieza hasta su posición definitiva (figura 9).

\section{COMPOSICIÓN}

La construcción está situada en una zona de carácter residencial de baja densidad (máximo dos plantas), por lo que el contraste con su entorno era inevitable.

Se ha optado por una solución que defina en conjunto unas formas geométricas claras, que predominen sobre la complejidad que forman visualmente las torres espaciales metálicas desde sus diversas perspectivas; los materiales y acabados, dentro de los condicionantes de carácter estructural, se han cuidado tratando de potenciar el carácter singular de la construcción dentro del conjunto en que está emplazada.

Con respecto a la diversidad de usos, contenida en el programa a desarrollar, se ha pretendido que quedaran integrados formalmente dentro del elemento estructural metálico que condiciona el aspecto de la construcción; los cerramientos de fábrica en la base, además de servir como zócalo a la torre, por su altura, similar a la de los edificios colindantes, se utilizan como único elemento de conexión con el entorno.

\section{RECONOCIMIENTO}

En el cálculo de la estructura colaboraron mis compañeros Enrique Gil Benso, Javier Ferranz Gonzálvez y Adolfo Alonso Durá, todos ellos de la Universidad Po. litécnica de Valencia.

\section{publicación del ICCET/CSIC \\ INSPECCION DE OBRAS DAÑADAS POR CORROSION DE ARMADURAS}

El presente Manual va dirigido principalmente a técnicos especializados y laboratorios que tienen que intervenir en el dictamen de la situación de deterioro de estructuras de hormigón armado dañadas por corrosión de armaduras.

Comienza con un resumen recordatorio de los factores principales a los que se pueden deber los daños prematuros por corrosión de armaduras, para seguir con algunas indicaciones de cómo se deben realizar las inspecciones, y de los ensayos y la metodología que se recomienda realizar para poder dictaminar con precisión las causas de daño.

A continuación se hacen una serie de comentarios sobre la vida residual de estructuras dañadas, sobre el riesgo de corrosión futura, el seguimiento necesario de una estructura reparada y una breve enumeración de métodos de reparación y consideraciones básicas a tener en cuenta en la recomendación de un determinado método. Se aporta una breve relación bibliográfica.

Finalmente se incluyen en forma de ficha la descripción de algunos casos de corrosión de armaduras detectados en nuestro país.

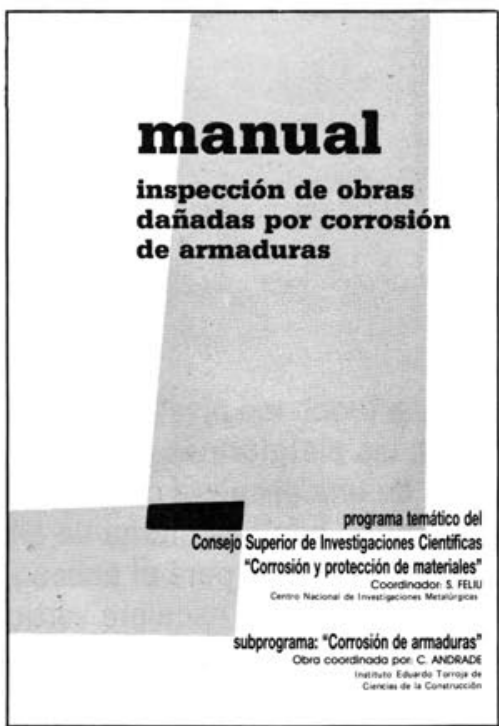

